\title{
TENSOR PRODUCTS OF POSITIVE DEFINITE QUADRATIC FORMS, VII
}

\section{YOSHIYUKI KITAOKA}

In this paper we generalize results of the third paper of this series. As a corollary we can show the following: Let $L_{i}(1 \leq i \leq n)$ be a positive definite quadratic form which is equivalent to one of Cartan matrices of Lie algebras of type $A_{n}(n \geq 2), D_{n}(n \geq 4), E_{6}, E_{7}, E_{8}$ and assume that $\otimes_{i=1}^{n} L_{i} \cong \otimes_{i=1}^{m} M_{i}$ where $M_{i}(1 \leq i \leq m)$ is positive definite quadratic forms and satisfies that $\operatorname{rk} M_{i} \geq 2$ and $M_{i} \cong K \otimes L$ implies $\operatorname{rk} K$ or $\operatorname{rk} L=1$. Then we have $n=m$ and $L_{i}$ is equivalent to a constant multiple of $M_{s(i)}$ for some permutation $s$. Therefore we get the uniqueness of decompositions with respect to tensor products in this case.

We explain notations and terminology.

By a positive lattice we mean a lattice on a positive definite quadratic space over the rational number field. Let $L$ be a positive lattice. We put

$$
m(L)=\min _{\substack{x \in L \\ x \neq 0}} Q(x),
$$

where $Q(\quad)$ is a quadratic form associated with $L$. Put $\mathfrak{M}(L)=\{x \in L \mid$ $Q(x)=m(L)\}$ and denote by $\tilde{L}$ a submodule of $L$ spanned by $\mathfrak{M}(L)$. If $\mathfrak{M}(L \otimes M) \subset\{x \otimes y \mid x \in L, y \in M\}$ holds for every positive lattice $M$, then $L$ is called of $E$-type and then $\mathfrak{M}(L \otimes M)=\mathfrak{M}(L) \otimes \mathfrak{M}(M), m(L \otimes M)=$ $m(L) m(M)$ hold. Unless $L$ is isometric to the tensor product of positive lattices $M, N$ with rk $M>1$, rk $N>1, L$ is called indecomposable with respect to tensor products.

Let $A$ be a finite set and [, ] a mapping from $A \times A$ to $\{t \mid 0 \leq t \leq 1\}$ satisfying

(i) $\left[a, a^{\prime}\right]=1$ if and only if $a=a^{\prime}$, and

(ii) $\left[a, a^{\prime}\right]=\left[a^{\prime}, a\right]$ for $a, a^{\prime} \in A$.

Then we call $(A,[]$,$) or simply A$ a weighted graph.

Received November 18, 1983. 
Let $A$ be a weighted graph. $A$ is called connected unless there exist subsets $A_{1}, A_{2}$ of $A$ such that $A=A_{1} \cup A_{2}, A_{1} \cap A_{2}=\phi$ and $\left[a_{1}, a_{2}\right]=0$ for any $a_{i} \in A_{i}$.

Let $A, B$ be weighted graphs. For $(a, b),\left(a^{\prime}, b^{\prime}\right) \in A \times B$ we define $\left[(a, b),\left(a^{\prime}, b^{\prime}\right)\right]$ by $\left[a, a^{\prime}\right] \cdot\left[b, b^{\prime}\right]$. Then $A \times B$ becomes a weighted graph. If there exists a bijection $\sigma$ from $A$ on $B$ such that $\left[a, a^{\prime}\right]=\left[\sigma(a), \sigma\left(a^{\prime}\right)\right]$ $\left(a, a^{\prime} \in A\right)$, then we say that $A, B$ are isometric and write $\sigma: A \cong B$. Unless a weighted graph $A$ is isometric to $B \times C(|B|>1,|C|>1)$, we say that $A$ is indecomposable.

Let $L$ be a positive lattice with a bilinear form $B(),(B(x, x)=Q(x))$. Put $G(L)=\mathfrak{M}(L) \mid \pm$ and for $a, b \in G(L)$, put $[a, b]=|B(a, b)| / m(L)$. Then $G(L)$ becomes a weighted graph.

Let $L, M$ be positive lattices. Then it is obvious that the isometry $\sigma: L \cong M$ induces the isometry $\tilde{\sigma}: G(L) \cong G(M)$, and that $G(L \otimes M) \cong$ $G(L) \times G(M)$ if either $L$ or $M$ is of $E$-type.

Lemma 1. Let $A, A^{\prime}, B, C$ be weighted graphs and assume that $A=$ $\left\{e_{i}\right\}_{i=1}^{n}$ and $\sigma: A \times B \cong A^{\prime} \times C$. Take any element $b \in B$ and fix it. Define $f_{i} \in A^{\prime}, c_{i} \in C, g_{i j} \in A, b_{i j} \in B$ by

$$
\sigma\left(e_{i}, b\right)=\left(f_{i}, c_{i}\right) \text { and } \sigma\left(g_{i j}, b_{i j}\right)=\left(f_{i}, c_{j}\right) .
$$

Then we have $\left[e_{i}, e_{j}\right]=0$ if $b_{i j} \neq b$.

Lemma 2. Let $A, A^{\prime}, B, C$ be weighted graphs and assume that $A=$ $\left\{e_{i}\right\}_{i=1}^{n}$ is connected and $\sigma: A \times B \cong A^{\prime} \times C$. Take any element $b \in B$ and put $\sigma\left(e_{i}, b\right)=\left(f_{i}, c_{i}\right)$. Then we have

$$
A \cong\left\{\sigma\left(e_{i}, b\right) \mid 1 \leq i \leq n\right\}=\left\{f_{i} \mid 1 \leq i \leq n\right\} \times\left\{c_{i} \mid 1 \leq i \leq n\right\} .
$$

Lemmas 1, 2 are proved in Section 1 of [4] when $A=A^{\prime}$. Moreover we did not use the condition $A=A^{\prime}$. Hence the proof in case of $A=A^{\prime}$ is valid with trivial changes like that $f_{i}$ is regarded as an element not of $A$ but of $A^{\prime}$.

Lemma 3. Let $A, B, C$ be connected weighted graphs and let $\sigma$ be an isometry from $A \times B$ on $A \times C$. If there exist $b_{0} \in B, c_{0} \in C$ such that $\sigma\left(x, b_{0}\right)=\left(f(x), c_{0}\right)$ for every $x \in A$, then $f$ is an isometry from $A$ on $A$ and there is an isometry $g$ from $B$ on $C$ with $\sigma(x, y)=(f(x), g(y))(x \in A, y \in B)$.

This is proved in [3]. 
Theorem. Let $A_{i}(1 \leq i \leq n), B_{i}(1 \leq i \leq m)$ be connected weighted graphs and suppose that $\left|A_{i}\right|>1,\left|B_{j}\right|>1$ and $A_{i}, B_{j}$ are indecomposable $(1 \leq i \leq n, 1 \leq j \leq m)$. Assume

$$
\sigma: \prod_{i=1}^{n} A_{i} \cong \prod_{i=1}^{m} B_{i}
$$

Then we have $n=m$ and there exist a permutation $s$ and isometries $\sigma_{i}: A_{i}$ $\cong B_{s(i)}$ and $\sigma$ is equal to the product of $\sigma_{i}$.

Proof. Without loss of generality we may assume that $\left|A_{1}\right| \geq\left|A_{i}\right|,\left|B_{j}\right|$ $(1 \leq i \leq n, 1 \leq j \leq m)$. Take any element $e_{i} \in A_{i}(i \geq 2)$ and put $e=e_{2}$ $\times \cdots \times e_{n} \in \prod_{i=2}^{n} A_{i}$. By interchanges of $B_{i}$ we may assume that the projection of $\sigma\left(A_{1} \times e\right)$ on $B_{1}$ includes at least two distinct elements. Applying Lemma 2 to $A=A_{1}, B=\prod_{i=2}^{n} A_{i}, A^{\prime}=B_{1}, C=\prod_{i=2}^{m} B_{\imath}$, we get

$$
\sigma\left(A_{1} \times e\right) \subset B_{1} \times c \quad \text { for some } c \in C,
$$

since $A_{1}$ is indecomposable. By the assumption on $A_{1}$ we have $\sigma\left(A_{1} \times e\right)$ $=B_{1} \times c$. Hence by virtue of Lemma 3 there exist isometries $f: A_{1} \cong B_{1}$, $g: \prod_{i=2}^{n} A_{i} \cong \prod_{i=2}^{m} B_{i}$ such that $\sigma(x \times y)=(f(x), g(y))$ for $x \in A_{1}, y \in \prod_{i=2}^{n} A_{i}$. Therefore our theorem is inductively proved.

TheOREM. Let $L_{i}(1 \leq i \leq n)$ be a positive lattice of $E$-type and assume that

(i ) $\left[L_{i}: \tilde{L}_{i}\right]<\infty$,

(ii) $\tilde{L}_{i}$ is indecomposable,

(iii) $L_{i}$ is indecomposable with respect to tensor products, and

(iv) $\operatorname{rk} L_{i}>1$.

Suppose that $\sigma: \bigotimes_{i=1}^{n} L_{i} \cong \bigotimes_{i=1}^{m} M_{i}$ where $M_{i}(1 \leq i \leq m)$ is a positive lattice satisfying the above conditions (iii), (iv) for $M_{i}$ instead of $L_{i}$. Then we have $n=m$ and, interchanging $M_{i}$ if necessary, $\sigma=\otimes \sigma_{i}$ where $\sigma_{i}$ is an isometry from $L_{i}$ on $M_{i}^{a_{i}}$ (scaling of $M_{i}$ by a positive constant $a_{i}$ ).

Proof. Put $L=\bigotimes_{i=1}^{n} L_{i}$. Since $L_{i}(1 \leq i \leq n)$ is of $E$-type, $L$ is also of $E$-type and $\tilde{L}=\bigotimes_{i=1}^{n} \tilde{L}_{i}$ and then $[L: \tilde{L}]<\infty$. Since $\tilde{L}_{i}$ is indecomposable and $\left[L_{i}: \tilde{L}_{i}\right]<\infty, L_{i}$ is also indecomposable. Hence $L$ and $\tilde{L}$ are indecomposable [2]. Then by virtue of Theorem in [4] $M_{i}$ is of $E$-type and satisfies the conditions (i) and (ii) for $M_{i}$ instead of $L_{i}$. Therefore without loss of generality we may assume that $m\left(L_{i}\right)=m\left(M_{j}\right)=1(1 \leq i \leq n$, $1 \leq j \leq m$ ) and interchanging $L_{i}$ if necessary, $L_{1}$ satisfies 
(1) $\operatorname{rk} L_{1} \geq \operatorname{rk} L_{i}$, rk $M_{j}(1 \leq i \leq n, 1 \leq j \leq m)$,

(2) if $\operatorname{rk} L_{1}=\operatorname{rk} M_{j}$, then $d L_{1} \leq d M_{j}$.

Since $\tilde{L}_{i}, \tilde{M}_{j}$ are indecomposable, associated graphs $G\left(L_{i}\right), G\left(M_{j}\right)$ are connected. $\sigma$ induces an isometry $\tilde{\sigma}: \prod G\left(L_{i}\right)=\prod G\left(M_{i}\right)$. Fix any element $e_{i} \in \mathfrak{M}\left(L_{i}\right)(i \geq 2)$ and $e_{i}$ is regarded as an element of $G\left(L_{i}\right)$. Then we have

$$
\tilde{\sigma}\left(G\left(L_{1}\right) \times e_{2} \times \cdots \times e_{n}\right)=\prod G_{i}, G_{i} \subset G\left(M_{i}\right) .
$$

Denoting by $M_{i}^{0}$ a submodule of $M_{i}$ spanned by elements of $\mathfrak{M}\left(M_{i}\right)$ which are projected in $G_{i}$, we get

$$
\sigma\left(\tilde{L}_{1} \otimes e_{2} \otimes \cdots \otimes e_{n}\right)=M_{1}^{0} \otimes \cdots \otimes M_{m}^{0} .
$$

Put $\bar{M}_{i}=M_{i} \cap \boldsymbol{Q} M_{i}^{0}$, then $\bar{M}_{i}$ is a direct summand of $M_{i}$ and $\left[\bar{M}_{i}: M_{i}^{0}\right]$ $<\infty$. Comparing direct summands, we have

$$
\sigma\left(L_{1} \otimes e_{2} \otimes \cdots \otimes e_{n}\right)=\bar{M}_{1} \otimes \cdots \otimes \bar{M}_{m} .
$$

By the assumption (iii) we may assume $\operatorname{rk} \bar{M}_{i}=1(i \geq 2)$, interchanging $M_{i}$ if necessary. Then $\bar{M}_{i}(i \geq 2)$ is spanned by an element $f_{i}$ in $\mathfrak{M}\left(M_{i}\right)$. Since we assumed $m\left(L_{i}\right)=m\left(M_{j}\right)=1$, there is an isometry $\sigma_{1}$ such that $\sigma\left(x \otimes e_{2} \otimes \cdots \otimes e_{n}\right)=\sigma_{1}(x) \otimes f_{2} \otimes \cdots \otimes f_{m}$. By virtue of Lemma 3 the isometry $\sigma_{1}$ is independent of $e_{i}(i \geq 2)$, since the sign can be absorbed in $f_{i}$. The assumption on $L_{1}$ implies $\operatorname{rk} M_{1} \leq \operatorname{rk} L_{1}=\operatorname{rk} \sigma_{1}\left(L_{1}\right) \leq \operatorname{rk} M_{1}$ and then $d L_{1}$ $\leq d M_{1} \leq d \sigma\left(L_{1}\right)=d L_{1}$ and then $\sigma_{1}\left(L_{1}\right)=M_{1}$. Moreover Lemma 3 implies that there is an isometry $\tilde{\sigma}_{2}: \prod_{i \geq 2} G\left(L_{i}\right) \cong \prod_{i \geq 2} G\left(M_{i}\right)$ such that $\tilde{\sigma}=\tilde{\sigma}_{1} \times \tilde{\sigma}_{2}$ on $\prod_{i=1}^{n} G\left(L_{i}\right)$. Therefore for any fixed $e_{1} \in \mathfrak{M}\left(L_{1}\right)$ we have

$$
\sigma\left(e_{1} \otimes e\right)=\sigma_{1}\left(e_{1}\right) \otimes \sigma_{2}(e) \quad \text { for } e \in \mathfrak{M}\left(\otimes_{i \geq 2} L_{i}\right),
$$

where $\sigma_{2}(e) \in \mathfrak{M}\left(\otimes_{i \geq 2} M_{i}\right)$.

Since $\sigma$ is an isometry, $\sigma_{2}$ is an isometry from $\otimes_{i \geq 2} \tilde{L}_{i}$ on $\otimes_{i \geq 2} \tilde{M}_{i}$. Moreover $\left[\otimes_{i \geq 2} L_{i}: \otimes_{i \geq 2} \tilde{L}_{i}\right]<\infty$ and $e_{1} \otimes\left(\otimes_{i \geq 2} L_{i}\right), \sigma_{1}\left(e_{1}\right) \otimes\left(\otimes_{i \geq 2} M_{i}\right)$ are direct summands. Hence $\sigma_{2}\left(\otimes_{i \geq 2} L_{i}\right)=\bigotimes_{i \geq 2} M_{i}$ follows. For $e_{1}^{\prime} \in \mathfrak{M}\left(L_{1}\right)$ we have, similarly, $\sigma\left(e_{1}^{\prime} \otimes e\right)=\sigma_{1}\left(e_{1}^{\prime}\right) \otimes \sigma_{2}^{\prime}(e)$ for $e \in \mathfrak{M}\left(\otimes_{i \geq 2} L_{i}\right)$ where $\sigma_{2}^{\prime}$ is an isometry from $\otimes_{i \geq 2} L_{i}$ on $\otimes_{i \geq 2} M_{i}$. Since $\tilde{\sigma}=\tilde{\sigma}_{1} \times \tilde{\sigma}_{2}, \sigma_{2}(e)= \pm \sigma_{2}^{\prime}(e)$ holds for $e \in \mathfrak{M}\left(\otimes_{i \geq 2} L_{i}\right)$. If $B\left(e_{1}, e_{1}^{\prime}\right) \neq 0$, then $B\left(e_{1}, e_{1}^{\prime}\right)=B\left(e_{1} \otimes e, e_{1}^{\prime} \otimes e\right)=B\left(\sigma_{1}\left(e_{1}\right)\right.$ $\left.\otimes \sigma_{2}(e), \sigma_{1}\left(e_{1}^{\prime}\right) \otimes \sigma_{2}^{\prime}(e)\right)=B\left(e_{1}, e_{1}^{\prime}\right) B\left(\sigma_{2}(e), \sigma_{2}^{\prime}(e)\right)$ implies $B\left(\sigma_{2}(e), \sigma_{2}^{\prime}(e)\right)=1$ and then $\sigma_{2}(e)=\sigma_{2}^{\prime}(e)$ since $m\left(M_{i}\right)=1$ and $\sigma_{2}(e), \sigma_{2}^{\prime}(e) \in \mathfrak{M}\left(\otimes_{i \geq 2} M_{i}\right)$. Thus $\sigma_{2}=\sigma_{2}^{\prime}$ holds on $\mathfrak{M}\left(\otimes_{i \geq 2} L_{i}\right)$ and then $\sigma_{2}=\sigma_{2}^{\prime}$ on $\otimes_{i \geq 2} L_{i}$. Since $G\left(L_{1}\right)$ is connected, 
$\sigma_{2}$ is independent of the choice of $e_{1}$. Thus we have proved $\sigma=\sigma_{1} \otimes \sigma_{2}$ on $\otimes_{i \geq 1} L_{i}$ where $\sigma_{1}: L_{1} \cong M_{1}, \sigma_{2}: \bigotimes_{i \geq 2} L_{i}=\bigotimes_{i \geq 2} M_{i}$. Theorem is inductively proved.

Corollary. Let $L_{i}$ be those in Theorem. Then the orthogonal group of $\otimes L_{i}$ is generated by the orthogonal group of $L_{i}(1 \leq i \leq n)$ and interchanges of $L_{i}$ and $L_{j}$ if $L_{i}, L_{j}$ are isometric.

This follows directly from Theorem.

Examples. Let $L$ be a positive lattice which is associated to the Cartan matrix of one of Lie algebras of type $A_{n}(n \geq 2), D_{n}(n \geq 4), E_{6}$, $E_{7}, E_{8}$. Then $L$ is of $E$-type [1] and the conditions (i), (ii), (iv) are obviously satisfied. The condition (iii) is checked as follows: Suppose $L \cong M \otimes N$, rk $M, \operatorname{rk} N>1$. Then by virtue of Theorem in [4], $M, N$ are of $E$-type and $M=\tilde{M}, N=\tilde{N}$ since $L=\tilde{L}$. Without loss of generality we may assume $m(M)=1, m(N)=2$. For $e \in \mathfrak{M}(M), f_{1}, f_{2} \in \mathfrak{M}(N), \quad Z \ni B\left(e \otimes f_{1}\right.$, $\left.e \otimes f_{2}\right)=B\left(f_{1}, f_{2}\right)$ follows. If $B\left(f_{1}, f_{2}\right)$ is even for every $f_{1}, f_{2} \in \mathfrak{M}(N)$, then $N$ is decomposable since the scale of $N$ is $2 Z$. This is a contradiction. Hence for some $f_{1}, f_{2} \in \mathfrak{M}(N), B\left(f_{1}, f_{2}\right)=1$ holds. Then we have $Z \ni B\left(e_{1} \otimes f_{1}\right.$, $\left.e_{2} \otimes f_{2}\right)=B\left(e_{1}, e_{2}\right)$ for $e_{i} \in \mathfrak{M}(M)$ and then the scale of $M$ is $Z$. Therefore $M$ is decomposable. This is also a contradiction.

Other examples are found in [4].

\section{REFERENCES}

[1] Y. Kitaoka, Scalar extension of quadratic lattices II, Nagoya Math. J., 67 (1977), 159-164.

[ 2 ] — - Tensor products of positive definite quadratic forms, Göttingen Nachr. Nr., 4 (1977).

[ 3 ] — - Tensor products of positive definite quadratic forms III, Nagoya Math. J., 70 (1978), 173-181.

[4] — Tensor products of positive definite quadratic forms, V, Nagoya Math. J., 82 (1981), 99-111.

[5] O. T. O'Meara, Introduction to quadratic forms, Berlin-Heidelberg-New York, 1963.

Department of Mathematics

Faculty of Science

Nagoya University

Chikusa-ku, Nagoya 464

Japan 